\title{
Severe Immune Thrombocytopenic Purpura Treated with Plasma Exchange
}

\author{
Sigdel M R, Shah D S, Kafle M P, Raut K B
}

Department of Internal Medicine, TU Teaching Hospital, Kathmandu, Nepal.

Corresponding Author

Mahesh Raj Sigdel

Department of Internal Medicine, TU Teaching

Hospital, Kathmandu, Nepal

Email:maheshsigdel@hotmail.com

Citation

Sigdel M R, Shah D S, Kafle M P, Raut K B. Severe Immune Thrombocytopenic Purpura Treated with Plasma Exchange. Kathmandu Univ Med J 2012;37(1):85-7.

\begin{abstract}
Immune thrombocytopenic purpura (ITP) is a hematological disorder characterized by immunologically mediated destruction of platelets and absence of other causes of thrombocytopenia. Treatment is required when the low platelet count entails risk of serious bleeding. Steroid is the first line of management. Acute refractory ITP with very low platelet count is variably treated with high dose steroid, intravenous immunoglobulin (IVIg), anti D or emergency splenectomy. Here, we present a case of steroid resistant ITP with severe thrombocytopenia treated with plasma exchange and low dose IVIg who responded dramatically to the therapy with maintained platelet count till one month from the institution of therapy.
\end{abstract}

\section{KEY WORDS}

immune thrombocytopenic purpura, intravenous immunoglobulin, plasma exchange

\section{INTRODUCTION}

Immune or idiopathic thrombocytopenic purpura (ITP) is an autoimmune disorder characterized by persistent thrombocytopenia, defined as a peripheral blood platelet count less than $100 \times 10^{9} / \mathrm{L}$, due to autoantibody binding to platelet antigen(s) causing their premature destruction by the system, and in particular the spleen. ${ }^{1,2}$ There is an associated impaired platelet production and $T$ cellmediated effects. ${ }^{3}$ There is no "gold standard" test that can reliably establish the diagnosis. ${ }^{4}$ The essential elements for the diagnosis include an otherwise healthy individual who presents with isolated thrombocytopenia, an otherwise unremarkable peripheral smear, a physical examination that only shows evidence of bleeding consistent with the platelet count, and the exclusion of other causes of thrombocytopenia. ${ }^{5}$ Specialized assays in the diagnosis of ITP include assays for anti-platelet antibodies, thrombopoietin (TPO) assays, reticulated platelets etc. ${ }^{6}$ However, their use in a routine case of ITP is not established. ITP is classified by duration into newly diagnosed, persistent (3-12 months' duration) and chronic (>12 months' duration). ${ }^{?}$

In general, patients with platelet counts exceeding $30 \times 10^{9}$ per litre require no treatment unless they are undergoing any procedure likely to induce blood loss. Prednisolone (or prednisone) is the standard initial first- line therapy for ITP patients who require treatment. ${ }^{4} \mathrm{High}$ dose dexamethasone or high dose intravenous methylprednisolone are used in severe, acute or refractory ITP. The alternative first line treatment of ITP include intravenous immunoglobulin (IVIG) or anti-D (WinRho)., The mechanism of action of IVIg in ITP remains largely unknown but is believed to involve the blockade of $\mathrm{Fc}$ receptors on macrophages and other effectors of antibody-dependent cytotoxicity, the presence of anti-idiotype antibodies in IVIg which block autoantibody binding to circulating platelets and immune suppression. ${ }^{6}$ Intravenous anti-D is appropriate for $\mathrm{Rh}(\mathrm{D})$ positive, non-splenectomized ITP patients and is avoided in autoimmune hemolytic anemia. Combining first-line therapies is appropriate in emergency settings eg steroid and IVIg. Other therapies that work rapidly include platelet transfusion, possibly in combination with IVIg, and emergency splenectomy. The second line therapies for persistent and chronic ITP include splenectomy, rituximab, danazol, TPO receptor agonists, vinca alkaloids, azathioprine, cyclosporin, cyclophosphamide, mycophenolate mofetil, dapsone etc, the choice of one agent over other depends on case to case basis. ${ }^{4,8-10}$

Plasmapheresis has been used by different people for the treatment of ITP with variable success. ${ }^{6}$ The general consensus is that plasmapheresis as an isolated approach is not justified in the treatment of ITP. ${ }^{4}$ We present a case of acute severe ITP treated primarily with plasma exchange followed by single low dose IVIg along with continuation of steroid. 


\section{CASE REPORT}

A 70 years lady, married, housewife, was referred to our hospital with complaint of sudden onset of reddish rashes all over the body, epistaxis and gum bleeding. She had history of three episodes of small volume loose motions one day prior to the onset of present symptoms, however she did not have fever, pain abdomen, blood in stool, tenesmus, running nose, sore throat, myalgia or arthralgia. She did not have cough, shortness of breath or chest pain. Her bladder habits were normal. She did not have bony pain anywhere, no localized lumps or swelling; there was no history of Raynaud's phenomenon, no jaundice in the past and no high risk behavior or exposure to blood or blood products prior to this episode. Her past history was significant for hypertension of two and half years' duration for which she was regularly taking amlodipine $2.5 \mathrm{mg} /$ day; apart from this she did not have exposure to any drugs, chemicals or radiation. Examination revealed pulse rate of $104 /$ minute, blood pressure of $160 / 70 \mathrm{mmHg}$, petechiae, purpura and ecchymoses all over the body and evidence of gum and lower lip bleeding; rest of the examination was normal. She did not have anemia, lymphadenopathy, bony tenderness or hepatosplenomegaly.

She had first presented to a medical college hospital on the same day of onset of symptoms where the platelet count was $1,000 / \mathrm{cmm}$ with total leukocyte count $6,600 / \mathrm{cmm}$, hemoglobin $9.2 \mathrm{gm} \%$ and normal peripheral smear. Her blood sugar, liver function tests and kidney function tests were normal. She was treated with Inj methyl prednisolone $1 \mathrm{gm}$ iv daily for three days from day one of presentation followed by oral prednisolone $60 \mathrm{mg} /$ day. In the five days before she presented to our hospital she had received 20 units of platelet rich plasma (PRP) and two pints of fresh blood. Her blood group was $\mathrm{O}$ positive.

The first reports at our hospital revealed hemoglobin 7.6 gm\%, leukocyte count $6200 / \mathrm{cmm}$ and platelet $4000 / \mathrm{cmmm}$, reticulocytes $2.5 \%$, normal peripheral smear otherwise, no evidence of fragmented RBCs, the biochemistry reports were normal except for indirect hyperbilirubinemia. Her HIV, $\mathrm{HBsAg}$, anti HCV serology was negative, ANA, anti DsDNA and VDRL were negative, coomb's test and anti-cardiolipin antibody tests were negative. Thyroid function test (TFT) was normal; bone marrow did not reveal malignant cells or any evidence of myelodysplastic syndrome. Steroid was continued first as oral prednisolone $60 \mathrm{mg} /$ day followed by Inj dexamethasone $8 \mathrm{mg}$ three times a day. On the third day of presentation she had upper gastrointestinal (UGI) bleeding evident as melena; UGI endoscopy revealed hemorrhagic gastritis. In the subsequent four days she received further 13 units of PRP with platelet at $3000 / \mathrm{cmm}$.

On the fifth day she underwent emergent therapeutic plasma exchange (TPE) via femoral double lumen catheter with Fresenius hemodialysis machine and standard plasma filter with 2.5 liters exchange volume $(1600 \mathrm{ml}$ 5\% human albumin and $900 \mathrm{ml}$ fresh frozen plasma, FFP) over two hours duration. Same day, two hours after TPE the platelet count rose to $43,000 / \mathrm{cmm}$ which dropped to $34,000 / \mathrm{cmm}$ the next day. With one day gap, second session of TPE was done following which platelet rose to $40,000 / \mathrm{cmm}$. Third TPE was done after two days of second session using 2 liters of $5 \%$ albumin and $600 \mathrm{ml} \mathrm{FFP.} \mathrm{Third} \mathrm{TPE} \mathrm{was} \mathrm{followed}$ by infusion of $5 \mathrm{gm}$ of intravenous immunoglobulin (IVIg). On next day of third TPE and IVIg, her platelet was 56,000/ $\mathrm{cmm}$ and $80,000 / \mathrm{cmm}$ the next day. Her subsequent serial platelet counts were $130,000 /$ and $140,000 / \mathrm{cmm}$ when she was discharged on 15th day of admission. During the entire stay she was given pantoprazole, amlodipine and prophylactic antibiotic coverage during TPE. She was discharged on prednisolone $40 \mathrm{mg} /$ day. Three days after discharge, the platelet count was $240,000 / \mathrm{cmm}$. Her platelet count was $312,000 / \mathrm{cmm}$ at three weeks after discharge.

\section{DISCUSSION}

In ITP, pathogenic autoantibodies bind platelets, resulting in accelerated platelet clearance. Targets of antiplatelet antibodies include glycoproteins IIb/IIIa and Ib/IX on the platelet membrane, although antibodies are demonstrable in only two-thirds of patients. ${ }^{3}$ Logically the therapy of ITP includes:

\section{- Suppression of antibody production}

- $\quad$ Suppression of clearance of the antibody bound platelets

- Removal of the main site of platelet destruction

- Stimulation of platelet production by the marrow

- Removal of the pathogenic autoantibodies or immune complexes. ${ }^{11}$

This makes the rationale for the use of various first and second line therapies in ITP. To date there have been few randomized controlled trials conducted in the management of ITP. Treatment should be tailored to the individual patient.

Some two-thirds of patients will respond to prednisolone at $1 \mathrm{mg} / \mathrm{kg}$ body weight per day, response appearing at 2-4 weeks. ${ }^{6}$ Our patient had acute and severe ITP, the platelet count was persistently below $4000 / \mathrm{cmm}$ at $10^{\text {th }}$ day of methyl prednisolone with evidence of active mucocutaneous and UGI bleeding that necessitated emergent therapy. The prohibitively high cost of full dose IVIg in our set up and the low hemoglobin and indirect hyperbilirubinemia prompted us to consider plasma exchange as an alternative and cheaper rescue therapy for our patient. Though splenectomy has a durable response rate of over $60 \%$, being an acute ITP, we ruled out the possibility of rescue emergency splenectomy in our patient. ${ }^{4}$ The increase in platelet count from $3000 / \mathrm{cmm}$ to $43,000 / \mathrm{cmm}$ after the first session of plasma exchange led us to give second exchange as well. These two exchanges 
stabilized the platelet to safe level of above $30,000 / \mathrm{cmm}$ for a few days. The proven response to plasma exchange in our patient and the recommendation for IVIg in acute severe ITP made us use low dose IVIg at the completion of third TPE after which platelet level rose up and was sustained.

However, a few questions remain unanswered in this patient. First, since she had acute ITP, the disease could have been going into self-remission by the third week, though spontaneous remission in adult ITP is not common. Second, she had been continued on high dose of steroid throughout the period of TPE when the response of steroid might have started. However, the immediate rise in platelet count after first session of TPE largely ruled out this possibility. Third, the low dose IVIg after third exchange could have significantly contributed to the further rise in platelet count. Fourth and the most important of all whether the rise in platelet with aforementioned therapies in our patient will result in sustained maintenance of platelet in normal level is yet to be seen. However, we believe that TPE rescued this patient from life threatening low platelet count and could be useful in similar set of circumstances if judiciously applied.

TPE, though not proven to be of much usefulness in the management of chronic ITP, could be a potentially lifesaving adjunctive approach in selected cases of acute severe ITP.

\section{REFERENCES}

1. Woods Jr, Kurata VL, Montgomery Y. Autoantibodies against platelet glycoprotein Ib in patients with chronic immune thrombocytopenic purpura. Blood 1984;64:156-60.

2. Woods VL, Oh EH, Mason D. Autoantibodies against the platelet glycoprotein IIb/IIla complex in patients with chronic ITP. Blood 1984; 63:368-75.

3. Fgarty PF, Minichiello T. Disorders of hemostasis, thrombosis and antithrombotic therapy. In: McPhee SJ, Papadakis MA, (editors), 2011. Current Medical Diagnosis \& Treatment. Mc Graw Hill 2011; 515-539.

4. Drew P, Stasi R, Newland CA. International consensus report on the investigation and management of primary immune thrombocytopenia. Blood 2010;115:168-86.

5. Cines DB, Bussel JB. How I treat idiopathic thrombocytopenic purpura (ITP). Blood 2005; 106:2244-51 .

7. Rodeghiero F, Stasi R, Gernsheimer T. Standardization of terminology, definitions and outcome criteria in immune thrombocytopenic purpura of adults and children: report from an international working group. Blood 2009;113(11):2386-93.

8. Arnold DM. Systematic review: efficacy and safety of rituximab for adults with idiopathic thrombocytopenic purpura. Ann Intern Med 2007 Jan;146(1):25-33.

9. Bussel JB. Eltrombopag for the treatment of chronic idiopathic thrombocytopenic purpura. N Engl J Med 2007 Nov;357(22):2237-47.

10. Kuter DJ. Efficacy of romiplostim in patients with chronic immune thrombocytopenic purpura: a double-blind randomised controlled trial. Lancet 2008 Feb;371(9610):395-403.

11. Barbara A. Konkle Disorders of platelets and vessel wall. In: Fauci $A$ S, Braunwald E, Kasper D L, editors. Harrisson's principles of internal medicine. Mc Graw Hill 2008; 718-725.

6. Guidelines for the investigation and management of idiopathic thrombocytopenic purpura in adults, children and in pregnancy. British Journal of Haematology 2003;120:574-96. 